ESAIM: COCV 23 (2017) 1129-1143

DOI: $10.1051 / \mathrm{cocv} / 2016026$
ESAIM: Control, Optimisation and Calculus of Variations

www.esaim-cocv.org

\title{
QUANTUM HAMILTONIAN AND DIPOLE MOMENT IDENTIFICATION IN PRESENCE OF LARGE CONTROL PERTURBATIONS
}

\author{
Ying Fu ${ }^{1}$ AND GABRIEL TURINICI ${ }^{1,2}$
}

\begin{abstract}
The problem of recovering the Hamiltonian and dipole moment is considered in a bilinear quantum control framework. The process uses as inputs some measurable quantities (observables) for each admissible control. If the implementation of the control is noisy the data available is only in the form of probability laws of the measured observable. Nevertheless it is proved that the inversion process still has unique solutions (up to phase factors). Both additive and multiplicative noises are considered. Numerical illustrations support the theoretical results.
\end{abstract}

Mathematics Subject Classification. 93-XX, 49-XX, 81Q93.

Received September 26, 2014. Revised January 20, 2016. Accepted May 17, 2016.

\section{INTRODUCTION AND MOTIVATION}

Successful manipulation of quantum dynamics (see [5] and references therein for a recent review) leads to interesting perspectives among which is the possibility to identify the system through measurements of controldependent observations. This technique, called quantum identification or quantum inversion, was documented both theoretically $[1,4,16,23]$ and numerically $[8,11,18]$. However although the numerical implementations show interesting robustness of the identification process with respect to noise, there is less theoretical guidance to explain this fact. Two fundamental questions concerning the well-posedness of this problem arise: the existence and the uniqueness of the Hamiltonian, and/or the dipole moment, compatible with the given measurements. In this work we only study the uniqueness.

More specifically we start from the setting in [16] which treats the case without noise. After some technical preliminaries in Section 3 we address the noise-free case in Section 4 and relax many of the assumptions used in the previous work. Then in Section 5 we introduce the possibility that the control is subject at each time to unknown perturbations. We consider both additive and multiplicative noise. Since the actual control that acts on the system is unknown, only the probability laws of the observations are available. We explain which are the properties of the set of measurements required to determine uniquely (up to phase factors) the free Hamiltonian and dipole moment.

Then a numerical implementation is presented in Section 6. Some closing remarks are the object of Section 7.

\footnotetext{
Keywords and phrases. Quantum control, quantum identification.

1 Université Paris-Dauphine, PSL Research University, CNRS, Ceremade, 75016 Paris, France. fu@ceremade.dauphine.fr

2 Institut Universitaire de France, 1 Rue Descartes, 75231 Paris cedex 05, France. Gabriel.Turinici@dauphine.fr
} 


\subsection{Notations}

We introduce the following notations

- $\mathbb{L}_{M_{1}, M_{2}, \ldots, M_{m}}$ is the Lie algebra spanned by the matrices $M_{1}, M_{2}, \ldots, M_{m}$;

- for any matrix or vector $X$ we denote by $\bar{X}$ its conjugate (the matrix whose entries are the complex conjugates of the entries of $X$ ) and by $X^{*}$ its adjoint (the transpose conjugate);

- $\mathcal{H}_{N}$ is the set of all Hermitian matrices $\mathcal{H}_{N}=\left\{X \in \mathbb{C}^{N \times N} \mid X^{*}=X\right\}$;

- $\mathcal{S}_{N}$ is the unit sphere of $\mathbb{C}^{N}: \mathcal{S}_{N}=\left\{v \in \mathbb{C}^{N} \mid\|v\|=1\right\}$;

- $\Psi\left(t, H, u(\cdot), \mu, \Psi_{0}\right)$ is the solution of the equation (2.1) below; to simplify the notation, when there is no ambiguity, we denote it $\Psi(t)$;

- $\lambda_{k}(X), k=1, \ldots, N$ are the eigenvalues of $X \in \mathcal{H}_{N}$ taken in increasing order; we also introduce $\phi_{k}(X)$ $k=1, \ldots, N$ to be eigenvectors of $X$ (forming an orthonormal basis of $\mathbb{C}^{N}$ ) corresponding to eigenvalues $\lambda_{k}(X)$; note that $\operatorname{Span}\left\{\phi_{k}(X)\right\}$ may not be unique;

- $S U(N)$ is the special unitary group of degree $N$, which is the group of $N \times N$ unitary matrices with determinant 1 ;

- $\mathfrak{s u}(N)$ is the Lie Algebra of skew-Hermitian matrices (the Lie algebra of $S U(N)$ );

\section{THE MODEL}

We present the mathematical framework following closely the notations of the previous work [16].

Consider a controlled quantum system with time-dependent wave-function $\Psi(t)$ satisfying the Schrödinger equation:

$$
\left\{\begin{array}{l}
i \dot{\Psi}\left(t, H, u(\cdot), \mu, \Psi_{0}\right)=(H+u(t) \mu) \Psi\left(t, H, u(\cdot), \mu, \Psi_{0}\right) \\
\Psi\left(0, H, u(\cdot), \mu, \Psi_{0}\right)=\Psi_{0},
\end{array}\right.
$$

where $H$ is the internal ("free") Hamiltonian and $\mu$ the coupling operator between the control $u(t) \in \mathrm{L}_{\text {loc }}^{1}\left(\mathbb{R}_{+} ; \mathbb{R}\right)$ and the system. We work in a finite dimensional framework, therefore $H, \mu \in \mathcal{H}_{N}$ for some $N \in \mathbb{N}^{*}$. The goal is to determine the matrix entries of $H$ and $\mu$ from laboratory measurements of some observables depending on $\Psi(t)$. The control $u(t)$ can be changed in order to gather enough information on the system.

However, contrary to [16], we allow in this work some time independent perturbations to appear in the control $u(t)$. That is, when the control is implemented in practice the nominal control intensity required by the experimentalist, denoted $\epsilon(t)$, is perturbed by $Y$ which means that $u=u(t, \epsilon(\cdot), Y)$; here $Y$ is a discrete random variable with possible outcomes $y_{1}, y_{2}, \ldots$. We assume that the law of the random variable $Y$ is time independent. A first example is the additive perturbation $u(t)=\epsilon(t)+Y$. Such perturbation models have already been used in the quantum computing literature under the name of "fixed systematic errors", see section VI.A. equation (40) of [14] or "systematic control error", see [15]. In [19] the authors use a noise model called "low frequency noise" (see Sect. IV.C. of [12]): it is defined as the portion of the (control) amplitude noise that has a correlation time that is long (up to $10^{3}$ times) compared to the timescale of the dynamics therefore it can be considered as constant in time. Additional noise models (additive or multiplicative) are presented in [24].

The perturbation $Y$ is unknown and thus $\Psi(t)$ is a random variable, as are all measurements depending on $\Psi(t)$. Repeating the control experiment several times the experimentalist will only learn the law of the measurements. From now on we will denote by $\mathcal{L}_{Y} Z$ the law of the random variable $Z$ (that is measurable with respect to the sigma-algebra generated by $Y$ ).

Two different settings are considered depending on which parameters are to be identified and the nature of the information available:

- Setting (S1): The Hamiltonian $H$ is known and the goal is to identify the dipole moment $\mu$.

- Setting (S2): Both the Hamiltonian $H$ and the dipole moment $\mu$ are unknown.

The measurements are of the form $\left\langle O \Psi\left(T, H, u, \mu, \Psi_{0}\right), \Psi\left(T, H, u, \mu, \Psi_{0}\right)\right\rangle$ with $O \in \mathcal{H}_{N}$ a member of a list of possible measurements. Often, the experimentalist only measures one observable in a list (but can repeat the 
experiment many times). This means that for general $O_{1}, O_{2} \in \mathcal{H}_{N}$ no information is available on the joint distribution of the values $\left\langle O_{1} \Psi\left(T, H, u, \mu, \Psi_{0}\right), \Psi\left(T, H, u, \mu, \Psi_{0}\right)\right\rangle$ and $\left\langle O_{2} \Psi\left(T, H, u, \mu, \Psi_{0}\right), \Psi\left(T, H, u, \mu, \Psi_{0}\right)\right\rangle$ of these two observables.

\section{SOME TECHNICAL PRELIMINARIES}

\subsection{Complete sets of commuting observables}

We recall in this section several facts about complete sets of commuting observables (hereafter abbreviated CSCO). We refer the reader to ([6], p. 146) for details.

First, recall that an observable is a self-adjoint operator on $\mathbb{C}^{N}$. Once a basis of $\mathbb{C}^{N}$ is chosen the observable can be represented as a matrix $O \in \mathcal{H}_{N}$.

A set of observables $\mathcal{O}=\left\{O_{1}, \ldots, O_{K}\right\}$ is called set of commuting observables (named SCO hereafter) if $\left[O_{k}, O_{\ell}\right]=0, \forall k, \ell \in\{1, \ldots, K\}$.

When all observables in the SCO are multiples of the identity operator the SCO is said to be trivial; unless specified otherwise, we only work with non-trivial SCO.

All observables in the SCO $\mathcal{O}$ can be diagonalized simultaneously i.e., there exists at least an orthonormal basis $\Phi=\left\{\phi_{1}, \ldots, \phi_{N}\right\}$ of $\mathbb{C}^{N}$ such that any $O \in \mathcal{O}$ is diagonal in the basis $\Phi$. This means that in particular any $\phi_{\ell}$ is an eigenvector of any observable $O \in \mathcal{O}$. In general the basis $\Phi$ is not unique because of possible degeneracies in the spectrum of the observables in $\mathcal{O}$. By definition a SCO is called a complete set of commuting observables (CSCO) if the orthonormal basis that diagonalizes the SCO is unique up to phase factors and permutations, i.e., if $\left\{\varphi_{1}, \ldots, \varphi_{N}\right\}$ is another orthonormal basis rendering all $O \in \mathcal{O}$ diagonal then there exists a permutation $\sigma$ of $\{1, \ldots, N\}$ and phases $\beta_{1}, \ldots, \beta_{N} \in \mathbb{R}$ such that $\varphi_{k}=\mathrm{e}^{i \beta_{k}} \phi_{\sigma(k)}$ for all $k=1, \ldots, N$.

Examples:

(1) Let $H$ be a Hamiltonian with all eigenvalues $\lambda_{\ell}(H)$ of multiplicity 1 . Then $\mathcal{O}=\{H\}$ is a CSCO.

(2) Let $\left\{v_{1}, \ldots, v_{N}\right\}$ be an orthonormal basis of $\mathbb{C}^{N}$. Then defining $P_{k}$ to be the projection on $v_{k}$ (that is $\left.P_{k}=v_{k} v_{k}^{*}\right)$ the set $\mathcal{O}=\left\{P_{k}, 1 \leq k \leq N\right\}$ is a CSCO. In this case $P_{k}$ are called populations of the states $v_{k}$.

(3) Consider $N=3$ and $\mathcal{O}=\left\{O_{d}\right\}$ with:

$$
O_{d}=\left(\begin{array}{ccc}
-1 & 0 & 0 \\
0 & 1 / 2 & 0 \\
0 & 0 & 1 / 2
\end{array}\right)
$$

Because the eigenspace corresponding to the eigenvalue $1 / 2$ is of dimension $2 \mathcal{O}$ is not a CSCO. In this case both the canonical base of $\mathbb{C}^{3}:\left\{(1,0,0)^{T},(0,1,0)^{T},(0,0,1)^{T}\right\}$ and the orthonormal basis $\left\{(1,0,0)^{T},(0,1 / 2,-\sqrt{3} / 2)^{T},(0, \sqrt{3} / 2,1 / 2)^{T}\right\}$ render $O_{d}$ diagonal.

(4) Consider the truncated spin-less Hydrogen atom whose eigenstates can be labeled by a set of three indexes $\phi_{n, l, m}$ with $n=1,2, \ldots, N_{t}, l=0,1, \ldots, n-1, m=-l,-l+1, \ldots, l-1, l$. Here $N_{t} \in \mathbb{N}$ is a fixed truncation threshold. A CSCO is given by the operators $H$ (Hamiltonian), $L^{2}$ (square of the angular momentum operator), $L_{z}$ (the $z$ component of the angular momentum operator) which act on the eigenstate $\phi_{n, l, m}$ as:

$$
H \phi_{n, l, m}=\frac{C_{H}}{n^{2}} \phi_{n, l, m}, L^{2} \phi_{n, l, m}=l(l+1) \hbar^{2} \phi_{n, l, m}, L_{z} \phi_{n, l, m}=m \hbar \phi_{n, l, m},
$$

with $C_{H}$ an universal constant and $\hbar$ the Plank constant. Here $n$ is called principal quantum number, $l$ the angular momentum quantum number and $m$ the magnetic quantum number. Note that in this case $\left\{H, L^{2}\right\}$ is a $\mathrm{SCO}$ but not a CSCO.

Measuring simultaneously all observables in a CSCO is in principle possible as it is compatible with the Heisenberg uncertainty principle since all observables in a CSCO commute two by two; therefore the values of those observables may be simultaneously computed with infinite precision. 
The following characterization of a $\mathrm{CSCO}$ will be used in the following sections:

Lemma 3.1. Let $\mathcal{O}=\left\{O_{1}, \ldots, O_{K}\right\}$ be a SCO. Then $\mathcal{O}$ is a CSCO iff there exist $\gamma_{1}, \ldots, \gamma_{K} \in \mathbb{R}$ such that all eigenvalues of $\sum_{k=1}^{K} \gamma_{k} O_{k}$ have multiplicity one.

Proof. We prove first the direct implication. Consider a basis $\Phi=\left\{\phi_{1}, \ldots, \phi_{N}\right\}$ of $\mathbb{C}^{N}$ that renders all $O_{k}$ diagonal and denote $\left(O_{k}\right)_{j}$ the $j$ th eigenvalue of $O_{k}$, that is $O_{k} \phi_{j}=\left(O_{k}\right)_{j} \phi_{j}$. Suppose now by contradiction that for any $\gamma=\left(\gamma_{1}, \ldots, \gamma_{K}\right) \in \mathbb{R}^{K}$ there exists $i(\gamma) \neq j(\gamma) \leq N$, such that $\sum_{k=1}^{K} \gamma_{k}\left(O_{k}\right)_{i(\gamma)}=\sum_{k=1}^{K} \gamma_{k}\left(O_{k}\right)_{j(\gamma)}$. Define the functions $g^{\ell_{1}, \ell_{2}}: \mathbb{R}^{K} \rightarrow \mathbb{R}$ by $g^{\ell_{1}, \ell_{2}}\left(\gamma_{1}, \ldots, \gamma_{K}\right)=\sum_{k=1}^{K} \gamma_{k}\left[\left(O_{k}\right)_{\ell_{1}}-\left(O_{k}\right)_{\ell_{2}}\right]$ and let $A^{\ell_{1}, \ell_{2}}=\{\gamma \in$ $\left.\mathbb{R}^{K} ; g^{\ell_{1}, \ell_{2}}(\gamma)=0\right\}$. We obtain that $\cup_{1 \leq \ell_{1}<\ell_{2} \leq N} A^{\ell_{1}, \ell_{2}}=\mathbb{R}^{K}$. By the Baire's theorem at least a couple $\left(\ell_{1}^{*}, \ell_{2}^{*}\right)$ exists such that $A^{\ell_{1}^{*}, \ell_{2}^{*}}$ has non empty interior. Therefore the analytic function $g^{\ell_{1}^{*}, \ell_{2}^{*}}$ is null on a non empty open set hence it is null everywhere.

But this means that $\left(O_{k}\right)_{\ell_{1}^{*}}=\left(O_{k}\right)_{\ell_{2}^{*}}$ for all $k=1, \ldots, K$. Therefore for all $O_{k} \in \mathcal{O}$ the $\ell_{1}^{*}$ th eigenvalue is of multiplicity 2 (the $\ell_{1}^{*}$ th eigenvector and the $\ell_{2}^{*}$ th eigenvector are associated to the same eigenvalue) which contradicts the uniqueness of the basis that diagonalizes $\mathcal{O}$, and hence we obtain a contradiction with the definition of a CSCO.

The reverse implication is more straightforward. Any basis $\Phi=\left\{\phi_{1}, \ldots, \phi_{N}\right\}$ that renders all $O_{k} \in \mathcal{O}$ diagonal will also render $\sum_{k=1}^{K} \gamma_{k} O_{k}$ diagonal. But by hypothesis all eigenvalues of $\sum_{k=1}^{K} \gamma_{k} O_{k}$ are distinct and therefore the basis $\Phi$ is unique (up to permutation and phases) and hence $\mathcal{O}$ is a CSCO.

\subsection{Background on controllability results}

Let $L \in \mathbb{N}^{*}$ and $G_{1}, \ldots, G_{L}$ be $L$ finite dimensional, connected, compact and simple Lie groups with the identity element $I d$. Let $A_{\ell}, B_{\ell} \in \mathfrak{g}_{\ell}$ for all $\ell=1, \ldots, L$ where $\mathfrak{g}_{\ell}$ is the Lie algebra of $G_{\ell}$.

Definition 3.2. Consider $L$ bilinear systems on the Lie groups $G_{\ell}$ :

$$
\left\{\begin{array}{l}
\frac{\mathrm{d} X_{\ell}(t)}{\mathrm{d} t}=\left(A_{\ell}+u(t) B_{\ell}\right) X_{\ell}(t), \\
X_{\ell}(0)=I d .
\end{array}\right.
$$

The systems are called simultaneously controllable (or ensemble controllable) if there exists $T_{A_{1}, \ldots, A_{L}, B_{1}, \ldots, B_{L}}>0$ such that for all $T \geq T_{A_{1}, \ldots, A_{L}, B_{1}, \ldots, B_{L}}$ and for all $V_{\ell} \in G_{\ell}, \ell=1, \ldots, L$ arbitrary, there exists a control $u \in \mathrm{L}^{1}([0, T], \mathbb{R})$ with $X_{\ell}(T)=V_{\ell}, \forall \ell=1, \ldots, L$.

Let $\mathcal{A}=A_{1} \oplus \ldots \oplus A_{L} \in \bigoplus_{\ell=1}^{L} \mathfrak{g}_{\ell}$ and $\mathcal{B}=B_{1} \oplus \ldots \oplus B_{L} \in \bigoplus_{\ell=1}^{L} \mathfrak{g}_{\ell}$. The following simultaneous controllability results are proved in ([21], Thms. $1 \& 2$ and [3], Lem. 3, p. 29).

Theorem 3.3. The collection (3.3) of L bilinear systems is simultaneously controllable if and only if $\mathbb{L}_{\mathcal{A}, \mathcal{B}}=$ $\bigoplus_{\ell=1}^{L} \mathfrak{g}_{\ell}$ or equivalently $\operatorname{dim}_{\mathbb{R}} \mathbb{L}_{\mathcal{A}, \mathcal{B}}=\sum_{\ell=1}^{L} \operatorname{dim}_{\mathbb{R}} \mathfrak{g}_{\ell}$.

Lemma 3.4. We suppose that $\mathbb{L}_{A_{\ell}, B_{\ell}}=\mathfrak{g}_{\ell}$, for all $\ell=1, \ldots, L$. Then $\mathbb{L}_{\mathcal{A}, \mathcal{B}} \neq \bigoplus_{\ell=1}^{L} \mathfrak{g}_{\ell}$ if and only if there exist $\ell, \ell^{\prime} \in\{1, \ldots, L\}, \ell \neq \ell^{\prime}$ and an isomorphism $f: \mathfrak{g}_{\ell} \rightarrow \mathfrak{g}_{\ell^{\prime}}$ such that $f\left(A_{\ell}\right)=A_{\ell^{\prime}}$ and $f\left(B_{\ell}\right)=B_{\ell^{\prime}}$.

Theorem 3.5. Let $G$ be a finite dimensional, connected, compact and simple Lie group and $\mathfrak{g}$ be its Lie algebra. Let $A, B \in \mathfrak{g}$ such that $\mathbb{L}_{A, B}=\mathfrak{g}$ and $\alpha_{1}, \ldots, \alpha_{L} \in \mathbb{R}$ be real constants, $\alpha_{i} \neq \alpha_{j} \forall i \neq j$. Consider the collection of control systems on $G$ :

$$
\left\{\begin{array}{l}
\frac{\mathrm{d} X_{\ell}(t)}{\mathrm{d} t}=\left\{A+\left(u(t)+\alpha_{\ell}\right) B\right\} X_{\ell}(t), \\
X_{\ell}(0)=I d .
\end{array}\right.
$$

Then the collection of systems (3.4) is simultaneously controllable. 
Remark 3.6. Although the Theorems 3.3 and 3.5 are formulated on a Lie group, this is enough to obtain controllability for the wave-function; recall that if $X(t, H, u(\cdot), \mu): \mathbb{R} \times \mathcal{H}_{N} \times \mathrm{L}_{\text {loc }}^{1}\left(\mathbb{R}_{+} ; \mathbb{R}\right) \times \mathcal{H}_{N} \rightarrow S U(N)$ satisfies the following equation:

$$
\left\{\begin{array}{l}
i \dot{X}(t, H, u(\cdot), \mu)=(H+u(t) \mu) X(t, H, u(\cdot), \mu) \\
X(0, H, u(\cdot), \mu)=I d
\end{array}\right.
$$

then $\Psi\left(t, H, u(\cdot), \mu, \Psi_{0}\right)=X(t, H, u(\cdot), \mu) \Psi_{0}$ is the solution of $(2.1)$. Since $S U(N)$ is transitive on the sphere $\mathcal{S}_{N}$ (see [7], p. 88), if the control system is controllable on the Lie group $S U(N)$ then it will also be controllable in the wave-function formulation.

\section{INVERSION WITHOUT NOISE}

Theorem 4.1 (Setting (S1)). Let $H, \mu_{1}, \mu_{2} \in \mathcal{H}_{N}, H$ diagonal, $\Psi_{0}^{1}, \Psi_{0}^{2} \in \mathcal{S}_{N}$ and denote for $a=1,2$ and $\epsilon \in \mathrm{L}_{\mathrm{loc}}^{1}\left(\mathbb{R}_{+}, \mathbb{R}\right): \Psi_{a}(t, \epsilon)=\Psi\left(t, H, \epsilon(\cdot), \mu_{a}, \Psi_{0}^{a}\right)$. Let $\mathcal{O}$ be a (non-trivial) $S C O$. We suppose that $N \geq 3$ and:

- (A1): $\mathbb{L}_{i H, i \mu_{1}}=\mathbb{L}_{i H, i \mu_{2}}=\mathfrak{s u}(N)$.

- (A2): $\operatorname{tr}(H)=\operatorname{tr}\left(\mu_{1}\right)=\operatorname{tr}\left(\mu_{2}\right)=0$.

- the eigenvalues of $H$ are all of multiplicity one.

Then there exists $T>0$ such that if:

$$
\left\langle O \Psi_{1}(T, \epsilon), \Psi_{1}(T, \epsilon)\right\rangle=\left\langle O \Psi_{2}(T, \epsilon), \Psi_{2}(T, \epsilon)\right\rangle \quad \forall \epsilon \in L^{1}([0, T] ; \mathbb{R}), \quad \forall O \in \mathcal{O},
$$

then for some $\left(\alpha_{i}\right)_{i=1}^{N} \in \mathbb{R}^{N}$ :

$$
\left(\mu_{1}\right)_{j k}=\mathrm{e}^{i\left(\alpha_{j}-\alpha_{k}\right)}\left(\mu_{2}\right)_{j k}, \forall j, k \leq N
$$

\section{Remark 4.2.}

(1) Assumption (A1) is required for the simultaneous controllability, see Theorem 3.3 and Lemma 3.4.

(2) The assumption (A2) can be made without loss of generality according to [16]. In fact, changing the Hamiltonian $H$ and/ or dipole moment $\mu$ by adding a multiple of the identity operator $I d$, does not change the observations. In this case, the state $\Psi(t)$ is replaced by $\mathrm{e}^{i \varphi} \Psi(t)$ with the phase $\varphi \in \mathbb{R}$ depending on $\operatorname{tr}(H), \operatorname{tr}(\mu)$ and on the control $u$.

Remark 4.3. The proof also shows that the values of any additional observable commuting with $H$ are identical for both systems, in particular all populations are always identical.

Moreover, when $\mu_{1}$ and $\mu_{2}$ are matrices of dipole operators (i.e., have the form of real potentials) truncated to dimension $N$, then $\mu_{1}$ and $\mu_{2}$ are real symmetric matrices; the Theorem implies $\left(\mu_{1}\right)_{j k}= \pm\left(\mu_{2}\right)_{j k}$ for all $j, k$. In general this is not enough to conclude that $\mu_{1}=\mu_{2}$ as it can be seen from the counter-example 1 from ([16], p. 381) where $N=3, \Psi_{0}^{1}=\Psi_{0}^{2}=(1,0,0)^{T}$ :

$$
H=\left(\begin{array}{ccc}
E_{1} & 0 & 0 \\
0 & E_{2} & 0 \\
0 & 0 & E_{2}
\end{array}\right), \quad \mu_{1}=\left(\begin{array}{ccc}
0 & -\mu_{\alpha} & 0 \\
-\mu_{\alpha} & 0 & \mu_{\beta} \\
0 & \mu_{\beta} & 0
\end{array}\right), \quad \mu_{2}=\left(\begin{array}{ccc}
0 & \mu_{\alpha} & 0 \\
\mu_{\alpha} & 0 & \mu_{\beta} \\
0 & \mu_{\beta} & 0
\end{array}\right), E_{1}, E_{2}, \mu_{\alpha}, \mu_{\beta} \in \mathbb{R} \text { (arbitrary). }
$$

In this case all control fields give rise to identical populations for both systems. This under-determination can be mitigated under additional hypothesis as in Remark 4.8 . 
Remark 4.4. When eigenvalues of $H$ are degenerate but $\mathcal{O}$ is a CSCO the Theorem 4.6 below should be used instead.

Proof. Consider the collection of two systems $\left(H, \mu_{1}\right)$ and $\left(H, \mu_{2}\right)$ seen as a control system on $S U(N) \bigoplus S U(N)$ with operators $i H \bigoplus i H, i \mu_{1} \bigoplus i \mu_{2} \in \mathfrak{s u}(N) \bigoplus \mathfrak{s u}(N)$. This collection can either be controllable or not. Denote $\mathcal{R}_{t}=\left\{\left(\Psi_{1}(t, \epsilon), \Psi_{2}(t, \epsilon)\right) \mid \epsilon \in L^{1}([0, t] ; \mathbb{R})\right\}, \mathcal{R}_{\infty}=\cup_{t \geq 0} \mathcal{R}_{t}$. It is known (see [13], Thm. 6.5 item (ii) p. 322) that there exists $T$ such that $\mathcal{R}_{T}=\mathcal{R}_{\infty}$.

Since $\mathcal{O}$ is a non-trivial SCO it contains at least an observable, denoted $O$, that is not multiple of the identity. For this observable there exist $\Psi_{x}, \Psi_{y} \in \mathcal{S}_{N}$ such that $\left\langle O \Psi_{x}, \Psi_{x}\right\rangle \neq\left\langle O \Psi_{y}, \Psi_{y}\right\rangle$. But the condition (4.1) shows that no control $\epsilon$ exists that drives $\Psi_{0}^{1}$ to $\Psi_{x}$ and $\Psi_{0}^{2}$ to $\Psi_{y}$; therefore the joint system $i H \bigoplus i H, i \mu_{1} \bigoplus i \mu_{2}$ is not controllable simultaneously. Then it exists an automorphism of $\mathfrak{s u}(N)$ that sends $i H$ to $i H$ and $i \mu_{1}$ to $i \mu_{2}$. But the automorphisms of $\mathfrak{s u}(N)$ are of the form $X \in \mathfrak{s u}(N) \mapsto W X W^{-1} \in \mathfrak{s u}(N)$ or $X \in \mathfrak{s u}(N) \mapsto W \bar{X} W^{-1} \in \mathfrak{s u}(N)$ for some $W \in S U(N)$. Recall that the matrix $H$ is real (because it is diagonal and in $\mathcal{H}_{N}$ ). Consider first that one can find $W \in S U(N)$ such that $H=W H W^{-1}$ and $\mu_{2}=W \mu_{1} W^{-1}$. The first identity shows that $[H, W]=0$ and therefore $W$ is diagonal with the diagonal containing entries of the form $\mathrm{e}^{i \alpha_{\ell}}, \ell \leq N$; the conclusion follows from the second identity. Consider now that there exists $W \in S U(N)$ such that $i H=W \overline{i H} W^{-1}$; then $\left[H^{2}, W\right]=0$, thus $W$ diagonal and therefore $H=-H$, impossible.

Remark 4.5. The result is stronger than the Theorem 1 in ([16], p. 380) which requires:

- A stronger condition on the spectrum of $H$ (the non-degenerate transition condition); recall that the transitions of $H$ are called non-degenerate if the eigenvalues $\lambda_{k}(H)$ of $H$ satisfy $\lambda_{i}(H)-\lambda_{j}(H) \neq \lambda_{a}(H)-\lambda_{b}(H)$ for all $(a, b) \neq(i, j)$. Here we only ask that the eigenvalues have multiplicity one.

- That observables in $\mathcal{O}$ are the populations (thus in particular $\mathcal{O}$ is a CSCO). Here a single non-trivial observable is enough.

- That the equality (4.1) take place at all times $T \geq 0$. Here only one time (large enough) is required.

Theorem 4.6 (Setting (S2)). Let $\mu_{1}, \mu_{2}, H_{1}, H_{2} \in \mathcal{H}_{N}, \Psi_{0}^{1}, \Psi_{0}^{2} \in \mathcal{S}_{N}$ and denote for $a=1,2$ and $\epsilon \in$ $\mathrm{L}_{\mathrm{loc}}^{1}\left(\mathbb{R}_{+}, \mathbb{R}\right): \Psi_{a}(t, \epsilon)=\Psi\left(t, H_{a}, \epsilon(\cdot), \mu_{a}, \Psi_{0}^{a}\right)$. We suppose that $N \geq 3$ and the following assumptions hold true:

(A1): $\mathbb{L}_{i H_{1}, i \mu_{1}}=\mathbb{L}_{i H_{2}, i \mu_{2}}=\mathfrak{s u}(N)$;

(A2): $\operatorname{tr}\left(H_{1}\right)=\operatorname{tr}\left(H_{2}\right)=\operatorname{tr}\left(\mu_{1}\right)=\operatorname{tr}\left(\mu_{2}\right)=0$;

Let $\mathcal{O}=\left\{O_{1}, \ldots, O_{K}\right\}$ be a CSCO and $\Phi=\left\{\phi_{1}, \ldots, \phi_{N}\right\}$ an orthonormal basis that diagonalizes $\mathcal{O}$.

Then there exists $T>0$ such that if:

$$
\left\langle O_{k} \Psi_{1}(T, \epsilon), \Psi_{1}(T, \epsilon)\right\rangle=\left\langle O_{k} \Psi_{2}(T, \epsilon), \Psi_{2}(T, \epsilon)\right\rangle \quad \forall \epsilon \in L^{1}([0, T] ; \mathbb{R}), \quad \forall k=1, \ldots, K,
$$

then there exist $\left(\alpha_{i}\right)_{i=1}^{N} \in \mathbb{R}^{N}$ and $\theta \in \mathbb{R}$ such that for all $j, k \leq N$ either

$$
\left.\left\langle\mu_{1} \phi_{j}, \phi_{k}\right\rangle=\mathrm{e}^{i\left(\alpha_{j}-\alpha_{k}\right)}\left\langle\mu_{2} \phi_{j}, \phi_{k}\right\rangle,\left\langle H_{1} \phi_{j}, \phi_{k}\right\rangle=\mathrm{e}^{i\left(\alpha_{j}-\alpha_{k}\right)}\left\langle H_{2} \phi_{j}, \phi_{k}\right\rangle,\left\langle\Psi_{0}^{1}, \phi_{j}\right\rangle=\mathrm{e}^{i\left(\theta-\alpha_{j}\right.}\right)\left\langle\Psi_{0}^{2}, \phi_{j}\right\rangle,
$$

or

$$
\left\langle\mu_{1} \phi_{j}, \phi_{k}\right\rangle=-\mathrm{e}^{i\left(\alpha_{j}-\alpha_{k}\right)} \overline{\left\langle\mu_{2} \phi_{j}, \phi_{k}\right\rangle}, \quad\left\langle H_{1} \phi_{j}, \phi_{k}\right\rangle=-\mathrm{e}^{i\left(\alpha_{j}-\alpha_{k}\right)} \overline{\left\langle H_{2} \phi_{j}, \phi_{k}\right\rangle},\left\langle\Psi_{0}^{1}, \phi_{j}\right\rangle=\mathrm{e}^{i\left(\theta-\alpha_{j}\right)} \overline{\left\langle\Psi_{0}^{2}, \phi_{j}\right\rangle} .
$$

Remark 4.7. When $\mathcal{O}$ is not a CSCO, the same proof allows only to obtain that an isomorphism of Lie algebras exists that sends $i H_{1}$ to $i H_{2}$ and $i \mu_{1}$ to $i \mu_{2}$. In general it is not possible to obtain more than a general isomorphism as shown by the following counter-example:

$$
H_{1}=\left(\begin{array}{ccc}
1 & 0 & 0 \\
0 & -1 / 2 & 0 \\
0 & 0 & -1 / 2
\end{array}\right), W=\left(\begin{array}{ccc}
1 & 0 & 0 \\
0 & 1 / 2 & \sqrt{3} / 2 \\
0 & -\sqrt{3} / 2 & 1 / 2
\end{array}\right), \quad \mu_{1}=\left(\begin{array}{lll}
0 & 1 & 2 \\
1 & 0 & 0 \\
2 & 0 & 0
\end{array}\right),
$$




$$
H_{2}=W H_{1} W^{-1}=H_{1}, \mu_{2}=W \mu_{1} W^{-1}=\left(\begin{array}{ccc}
0 & \sqrt{3}+1 / 2 & 1-\sqrt{3} / 2 \\
\sqrt{3}+1 / 2 & 0 & 0 \\
1-\sqrt{3} / 2 & 0 & 0
\end{array}\right), \Psi_{0}^{2}=W \Psi_{0}^{1}, \mathcal{O}=\left\{O_{d}\right\}
$$

It is immediate to see that $\left\langle O_{d} \psi, \psi\right\rangle=1 / 2-3 / 2\left|\left\langle\psi,(1,0,0)^{T}\right\rangle\right|^{2}$ and that $W(1,0,0)^{T}=(1,0,0)^{T}$. When the control $\epsilon$ on the first system realizes the transformation $X$ the observable is $1 / 2-3 / 2\left|\left\langle X \Psi_{0}^{1},(1,0,0)^{T}\right\rangle\right|^{2}$; at the same time the control realizes the transformation $W X W^{-1}$ on the second system giving the observable $1 / 2-$ $3 / 2\left|\left\langle W X W^{-1} W \Psi_{0}^{1},(1,0,0)^{T}\right\rangle\right|^{2}$. But $\left\langle W X W^{-1} W \Psi_{0}^{1},(1,0,0)^{T}\right\rangle=\left\langle X \Psi_{0}^{1}, W^{-1}(1,0,0)^{T}\right\rangle=\left\langle X \Psi_{0}^{1},(1,0,0)^{T}\right\rangle$. Therefore it is not possible to distinguish between the couple $\left(H_{1}, \mu_{1}\right)$ and $\left(H_{2}, \mu_{2}\right)$ (at least for this initial data).

Remark 4.8. If, for physical reasons, we know the initial state of the system, then $\Psi_{0}^{1}=\Psi_{0}^{2}$; when this initial state has non-zero components along every element of the basis, i.e. $\left\langle\Psi_{0}^{1}, \phi_{k}\right\rangle \neq 0$, for all $k \geq 1$ the equation (4.5) implies $\mathrm{e}^{i \alpha_{j}}=\mathrm{e}^{i \theta}$ for all $j=1, \ldots, N$ which means $H_{1}=H_{2}$ and $\mu_{1}=\mu_{2}$. When some coefficients $\left\langle\Psi_{0}^{1}, \phi_{k}\right\rangle$ are zero, further symmetries may occur and one can have, for instance, $\mu_{1} \neq \mu_{2}$ : see counter-example 1 from ([16], p. 381) presented in Remark 4.3.

On the other hand, in this case, the conclusion (4.6) can be written more conveniently in the adapted basis $\left\{v_{1}=\mathrm{e}^{-i \alpha_{1} / 2} \phi_{1}, \ldots, v_{N}=\mathrm{e}^{-i \alpha_{N} / 2} \phi_{k}\right\}$ :

$$
\left\langle\mu_{1} v_{j}, v_{k}\right\rangle=-\overline{\left\langle\mu_{2} v_{j}, v_{k}\right\rangle},\left\langle H_{1} v_{j}, v_{k}\right\rangle=-\overline{\left\langle H_{2} v_{j}, v_{k}\right\rangle}, \Psi_{0}^{1}=\Psi_{0}^{2}=\mathrm{e}^{i \theta / 2} \sum_{\ell=1}^{N} \varsigma_{\ell} v_{\ell}, \varsigma_{\ell} \in \mathbb{R} .
$$

Proof. Denote by $T$ the time at which the couple of systems $\left(H_{1}, \mu_{1}\right),\left(H_{2}, \mu_{2}\right)$, seen as a control system on $S U(N) \bigoplus S U(N)$ with operators $i H \bigoplus i H, i \mu_{1} \bigoplus i \mu_{2} \in \mathfrak{s u}(N) \bigoplus \mathfrak{s u}(N)$ reaches all attainable states. Since a CSCO is a non-trivial SCO it follows as in the Theorem 4.1 that there exists an isomorphism of $f: \mathfrak{s u}(N) \rightarrow$ $\mathfrak{s u}(N)$ such that $i H_{2}=f\left(i H_{1}\right), i \mu_{2}=f\left(i \mu_{1}\right)$.

All isomorphisms of $\mathfrak{s u}(N)$ are of the form $\mathfrak{X} \in \mathfrak{s u}(N) \mapsto W \mathfrak{X} W^{-1} \in \mathfrak{s u}(N)$ or $\mathfrak{X} \in \mathfrak{s u}(N) \mapsto W \overline{\mathfrak{X}} W^{-1} \in$ $\mathfrak{s u}(N)$ for some $W \in S U(N)$. We only treat here the "exotic" case $f(\mathfrak{X})=W \overline{\mathfrak{X}} W^{-1}$ as the second alternative is similar. Thus $H_{2}=-W \overline{H_{1}} W^{-1}$ and $\mu_{2}=-W \overline{\mu_{1}} W^{-1}$. With the notations in the equation (3.5) we write:

$$
X\left(t, H_{2}, u(\cdot), \mu_{2}\right)=X\left(t,-W \overline{H_{1}} W^{-1}, u(\cdot),-W \overline{\mu_{1}} W^{-1}\right)=W \overline{X\left(t, H_{1}, u(\cdot), \mu_{1}\right)} W^{-1} .
$$

As the first system is controllable then every state $X \in S U(N)$ can be reached by some control $u(\cdot)$ thus

$$
\left\langle O_{k} X \Psi_{0}^{1}, X \Psi_{0}^{1}\right\rangle=\left\langle O_{k} W \bar{X} W^{-1} \Psi_{0}^{2}, W \bar{X} W^{-1} \Psi_{0}^{2}\right\rangle, \forall X \in S U(N), \forall k \leq K .
$$

Note that (4.11) also holds for any linear combination of observables in $\mathcal{O}$. We invoke the Lemma 3.1 and obtain the existence of an observable $O$, diagonal in the basis $\Phi$ and with all eigenvalues distinct, such that

$$
\left\langle O X \Psi_{0}^{1}, X \Psi_{0}^{1}\right\rangle=\left\langle O W \bar{X} W^{-1} \Psi_{0}^{2}, W \bar{X} W^{-1} \Psi_{0}^{2}\right\rangle, \forall X \in S U(N) .
$$

The vectors $\phi_{k}$ are eigenvectors of $O$ and denote as $\lambda_{k}(O)$ the corresponding eigenvalues. In particular $O=\sum_{k=1}^{N} \lambda_{k}(O) \phi_{k} \phi_{k}^{*}$. We can suppose that $\lambda_{1}(O)<\lambda_{2}(O)<\ldots<\lambda_{N}(O)$ (otherwise re-index the vectors).

Let us write $\overline{W^{-1} \Psi_{0}^{2}}=x \Psi_{0}^{1}+y v$ with $x, y \in \mathbb{C},|x|^{2}+|y|^{2}=1, v \in \mathcal{S}_{N}, v \perp \Psi_{0}^{1}$.

Suppose $y \neq 0$; then there exists $X \in S U(N)$ such that $X \Psi_{0}^{1}=\phi_{N}$ and $X v \in \operatorname{Span}\left\{\overline{W^{-1} \phi_{N}}, \phi_{N}\right\}^{\perp}$. Then $\left\langle O X \Psi_{0}^{1}, X \Psi_{0}^{1}\right\rangle=\lambda_{N}(O)=\left\langle O W \bar{X} W^{-1} \Psi_{0}^{2}, W \bar{X} W^{-1} \Psi_{0}^{2}\right\rangle$. Since $\lambda_{N}(O)$ is the maximum possible value for $O$ and all eigenspaces of $O$ are of dimension 1 it follows that $W \bar{X} W^{-1} \Psi_{0}^{2} \in \operatorname{Span}\left\{\phi_{N}\right\}$ hence $X \overline{W^{-1} \Psi_{0}^{2}} \in$ $\operatorname{Span}\left\{\overline{W^{-1} \phi_{N}}\right\}$. Then:

$$
1=\left|\left\langle X \overline{W^{-1} \Psi_{0}^{2}}, \overline{W^{-1} \phi_{N}}\right\rangle\right|=\left|\left\langle X\left(x \Psi_{0}^{1}+y v\right), \overline{W^{-1} \phi_{N}}\right\rangle\right|=\left|\left\langle x \phi_{N}, \overline{W^{-1} \phi_{N}}\right\rangle\right|=|x|\left|\left\langle\phi_{N}, \overline{W^{-1} \phi_{N}}\right\rangle\right| .
$$


It follows $y=0,|x|=1$; therefore $\overline{W^{-1} \Psi_{0}^{2}} \in \operatorname{Span}\left\{\Psi_{0}^{1}\right\}$ which means that there exists $\theta \in \mathbb{R}$ such that $\Psi_{0}^{2}=\mathrm{e}^{i \theta} W \overline{\Psi_{0}^{1}}$; after trivial simplifications the equality (4.12) can be written

$$
\left\langle O X \Psi_{0}^{1}, X \Psi_{0}^{1}\right\rangle=\left\langle O W \overline{X \Psi_{0}^{1}}, W \overline{X \Psi_{0}^{1}}\right\rangle, \forall X \in S U(N) .
$$

But $X \in S U(N)$ means that $X \Psi_{0}^{1}$ can be chosen arbitrary in $\mathcal{S}_{N}$; we have therefore:

$$
\forall w \in \mathcal{S}_{N}:\langle O w, w\rangle=\langle O W \bar{w}, W \bar{w}\rangle=\left\langle W^{*} O W \bar{w}, \bar{w}\right\rangle=\left\langle\overline{W^{*} O W} w, w\right\rangle .
$$

But this implies $O=\overline{W^{*} O W}$ and thus:

$$
\sum_{k=1}^{N} \lambda_{k}(O) \phi_{k} \phi_{k}^{*}=O=\overline{W^{*} O W}=\overline{W^{*}\left(\sum_{k=1}^{N} \lambda_{k}(O) \phi_{k} \phi_{k}^{*}\right) W}=\sum_{k=1}^{N} \lambda_{k}(O)\left(\bar{W}^{*} \overline{\phi_{k}}\right)\left(\bar{W}^{*} \overline{\phi_{k}}\right)^{*} .
$$

Since all eigenvalues of $O$ are non-degenerate the representation $O=\sum_{k=1}^{N} \lambda_{k}(O) \phi_{k} \phi_{k}^{*}$ is unique up to phases. Therefore there exist $\alpha_{k} \in \mathbb{R}$ such that $\overline{W^{*}} \overline{\phi_{k}}=\mathrm{e}^{-i \alpha_{k}} \phi_{k}$ or, equivalently, $W^{*} \phi_{k}=\mathrm{e}^{i \alpha_{k}} \overline{\phi_{k}}$.

The conclusion follows from the relationships $H_{2}=-W \overline{H_{1}} W^{*}, \mu_{2}=-W \overline{\mu_{1}} W^{*}$ and $\Psi_{0}^{2}=\mathrm{e}^{i \theta} W \overline{\Psi_{0}^{1}}$.

\section{INVERSION IN PRESENCE OF NOISE}

Let $(\Omega, \mathcal{F}, \mathbb{P})$ be a discrete probability space, $\mathcal{V}=\left\{y_{\ell} \in \mathbb{R}^{d} \mid \ell \in \mathcal{I} \subset \mathbb{N}\right\}$ a set of values in $\mathbb{R}^{d}$ (possibly infinite) and let $Y: \Omega \rightarrow \mathcal{V}$ be a random variable. We can suppose that for all $y_{\ell} \in \mathcal{V}, \mathbb{P}\left(Y=y_{\ell}\right)>0$ (otherwise we eliminate all $y_{\ell}$ such that $\left.\mathbb{P}\left(Y=y_{\ell}\right)=0\right)$. Moreover after re-indexing $\mathcal{I}$ we can suppose that $\mathcal{I}=\mathbb{N}^{*}$ or $\mathcal{I}=\left\{1, \ldots, L_{0}\right\}$ for some $L_{0} \in \mathbb{N}^{*}$. Denote $\xi_{k}=\mathbb{P}\left(Y=y_{k}\right), \forall k \in \mathcal{I}$.

We can suppose that $\left(\xi_{\ell}\right)_{\ell \geq 1}$ is a decreasing sequence (re-indexing if necessary).

\subsection{Technical preliminaries: a correspondence lemma}

Let $J_{a}: \mathbb{C}^{N \times N} \rightarrow \mathbb{R}, a=1,2$ and $h: \mathbb{R}^{d+1} \rightarrow \mathbb{R}$ be real analytic functions with $J_{a}$ bounded.

Lemma 5.1. Let $A_{a}, B_{a} \in \mathfrak{s u}(N), T>0, \epsilon \in \mathrm{L}^{1}([0, T], \mathbb{R})$ and denote by $X_{a}\left(t, y_{\ell}, \epsilon\right)$ the solution of

$$
\left\{\begin{array}{l}
\frac{\mathrm{d} X_{a}\left(t, y_{\ell}, \epsilon\right)}{\mathrm{d} t}=\left(A_{a}+h\left(\epsilon(t), y_{\ell}\right) B_{a}\right) X_{a}\left(t, y_{\ell}, \epsilon\right) \\
X_{a}\left(0, y_{\ell}, \epsilon\right)=I d
\end{array}\right.
$$

for $a=1,2$ and any $\ell \in \mathcal{I}$. Suppose that the following equality in law holds

$$
\mathcal{L}_{Y}\left(J_{1}\left(X_{1}(T, Y, \epsilon)\right)\right)=\mathcal{L}_{Y}\left(J_{2}\left(X_{2}(T, Y, \epsilon)\right)\right) \quad \forall \epsilon \in \mathrm{L}^{1}([0, T], \mathbb{R})
$$

Then for any $\ell \in \mathcal{I}$, there exists $n_{0}\left(\ell, \xi_{1}, \ldots, \xi_{n}, \ldots\right)$ and $\kappa(\ell) \in \mathcal{I}, \kappa(\ell) \leq n_{0}\left(\ell, \xi_{1}, \ldots, \xi_{n}, \ldots\right)$ such that

$$
J_{1}\left(X_{1}\left(T, y_{\ell}, \epsilon\right)\right)=J_{2}\left(X_{2}\left(T, y_{\kappa(\ell)}, \epsilon\right)\right) \quad \forall \epsilon \in \mathrm{L}^{1}([0, T], \mathbb{R}) .
$$

Proof. Let $\ell \in \mathcal{I}$. The proof is divided in several steps.

\section{Step 1:}

Fix a control $\epsilon$. We introduce the notation:

$$
v_{a}^{k}=J_{a}\left(X_{a}\left(T, y_{k}, \epsilon\right)\right), \quad a=1,2 \quad \text { and } \quad k \in \mathcal{I} .
$$


According to the assumption (5.2) we know that $J_{1}\left(X_{1}(T, Y, \epsilon)\right)$ and $J_{2}\left(X_{2}(T, Y, \epsilon)\right)$ follow the same law. Thus $\mathbb{P}\left(J_{1}\left(X_{1}(T, Y, \epsilon)\right)=v_{1}^{\ell}\right)=\mathbb{P}\left(J_{2}\left(X_{2}(T, Y, \epsilon)\right)=v_{1}^{\ell}\right)$. Then

$$
\sum_{k^{\prime} \in \mathcal{I} / v_{2}^{k^{\prime}}=v_{1}^{\ell}} \xi_{k^{\prime}}=\sum_{k \in \mathcal{I} / v_{1}^{k}=v_{1}^{\ell}} \xi_{k} \geq \xi_{\ell}>0 .
$$

Therefore $\left\{k^{\prime} \in \mathcal{I} / v_{2}^{k^{\prime}}=v_{1}^{\ell}\right\} \neq \emptyset$. In addition, there exists a $n_{0}(\ell) \in \mathcal{I}$ such that $\sum_{k>n_{0}(\ell), k \in \mathcal{I}} \xi_{k}<\xi_{\ell}$ and $\sum_{k>n_{0}(\ell)-1, k \in \mathcal{I}} \xi_{k} \geq \xi_{\ell}$ (by convention a sum over an empty set of indexes is zero). So we have $\left\{k^{\prime} \in \mathcal{I} / k^{\prime} \leq\right.$ $\left.n_{0}(\ell), v_{2}^{k^{\prime}}=v_{1}^{\ell}\right\} \neq \emptyset$. The index $n_{0}(\ell)$ depends only on the law of $Y$ and the index $\ell$.

Letting $\epsilon$ vary in $L^{1}([0, T] ; \mathbb{R})$ we obtain a function $\kappa_{1}: L^{1}([0, T] ; \mathbb{R}) \rightarrow\left\{k \in \mathcal{I}, k \leq n_{0}(\ell)\right\}$ such that

$$
J_{1}\left(X_{1}\left(T, y_{\ell}, \epsilon\right)\right)=J_{2}\left(X_{2}\left(T, y_{\kappa_{1}(\epsilon)}, \epsilon\right)\right) .
$$

Note: when the index $\kappa_{1}(\epsilon)$ with the property $(5.5)$ is not unique, any compatible value in the set $\{k \in \mathcal{I}, k \leq$ $\left.n_{0}(\ell)\right\}$ can be chosen.

\section{Step 2:}

Let $n \in \mathbb{N}^{*}$. We consider the space $\mathcal{P}_{n}$ of piecewise constant controls $\mathcal{P}_{n}=\left\{f:[0, T] \rightarrow \mathbb{R} \mid \quad f=\alpha_{1} \mathbf{1}_{\left[0, \frac{T}{n}\right]}+\right.$ $\left.\alpha_{2} \mathbf{1}_{\left.] \frac{T}{n}, \frac{2 T}{n}\right]}+\ldots+\alpha_{n} \mathbf{1}_{\left.] \frac{(n-1)}{n} T, T\right]}, \quad \alpha_{1}, \ldots, \alpha_{n} \in \mathbb{R}\right\}$. Denote $\alpha=\left(\alpha_{1}, \ldots, \alpha_{n}\right)$. Therefore for any $k \in \mathcal{I}$, we can define the functions $g_{k}$ from $\mathbb{R}^{n}$ to $\mathbb{R}$ by

$$
g_{k}(\alpha)=J_{2}\left(X_{2}\left(T, y_{k}, \epsilon_{\alpha}\right)\right)-J_{1}\left(X_{1}\left(T, y_{\ell}, \epsilon_{\alpha}\right)\right),
$$

with $\epsilon_{\alpha}=\alpha_{1} \mathbf{1}_{\left[0, \frac{T}{n}\right]}+\alpha_{2} \mathbf{1}_{\left.] \frac{T}{n}, \frac{2 T}{n}\right]}+\ldots+\alpha_{n} \mathbf{1}_{\left.] \frac{(n-1)}{n} T, T\right]}$. We know that

$$
X_{a}\left(T, y_{k}, \epsilon_{\alpha}\right)=\mathrm{e}^{\left(A_{a}+h\left(\alpha_{n}, y_{k}\right) B_{a}\right) \frac{T}{n}} \mathrm{e}^{\left(A_{a}+h\left(\alpha_{n-1}, y_{k}\right) B_{a}\right) \frac{T}{n}} \ldots \mathrm{e}^{\left(A_{a}+h\left(\alpha_{1}, y_{k}\right) B_{a}\right) \frac{T}{n}} \quad a=1,2 .
$$

Therefore the functions $X_{a}$ are analytic in $\alpha$ (recall that the function $h$ is analytic in $\alpha$ ), and since $J_{a}$ are analytic, the functions $g_{k}$ are analytic. We denote $A_{k}=\left\{\alpha \in \mathbb{R}^{n} / g_{k}(\alpha)=0\right\}$. Each $A_{k}$ is closed because $g_{k}$ is continuous. In Step 1, it is proved that

$$
\exists \kappa^{\mathcal{P}}: \mathbb{R}^{n} \rightarrow\left\{k \in \mathcal{I}, k \leq n_{0}(\ell)\right\} \quad \text { such that } \forall \alpha \in \mathbb{R}^{n} \quad g_{\kappa^{\mathcal{P}}(\alpha)}(\alpha)=0 .
$$

So $\bigcup_{k \in \mathcal{I}, k \leq n_{0}(\ell)} A_{k}=\mathbb{R}^{n}$. By the Baire's theorem, it exists a $k$ such that $A_{k}$ has an interior point. This means that $g_{k}$ is analytic and identically zero on a not empty open set. Therefore, $g_{k} \equiv 0$. So $\forall n, \exists \kappa_{2}(n) \in\{k \in \mathcal{I}, k \leq$ $\left.n_{0}(\ell)\right\}$ such that $g_{\kappa_{2}(n)}(\epsilon)=0$, for any control $\epsilon \in \mathcal{P}_{n}$.

\section{Step 3:}

Take $q \in \mathbb{N}$ and denote $\left.B_{q}=\left\{k \in \mathcal{I}, k \leq n_{0}(\ell)\right\} / g_{k}(\epsilon)=0, \quad \forall \epsilon \in \mathcal{P}_{2^{q}}\right\}$. In Step 2 it is proved that for any $q \in \mathbb{N}$ the set $B_{q}$ is not empty. Obviously $\left(B_{q}\right)_{q \in \mathbb{N}}$ is a decreasing sequence and $B_{q}$ becomes constant from a certain term, thus $B_{\infty}=\bigcap_{q>0} B_{q} \neq \emptyset$. This means that there exists $\kappa(\ell) \in\left\{k \in \mathcal{I}, k \leq n_{0}(\ell)\right\}$ such that $g_{\kappa(\ell)}(\epsilon)=0, \forall \epsilon \in \mathcal{P}_{2^{q}}$ for all $q$. Yet, $\bigcup_{q=0}^{\infty} \mathcal{P}_{2^{q}}$ is dense in $L^{1}([0, T] ; \mathbb{R})$. So we have $g_{\kappa(\ell)}(\epsilon)=0$, for any control $\epsilon$ in $L^{1}([0, T] ; \mathbb{R})$.

\subsection{Main results}

We set $d=1$.

Theorem 5.2. Consider the same setting and assumptions as in the Theorem 4.6 with the exception of the relation (4.4). Then there exists $T>0$ such that if:

$$
\mathcal{L}_{Y}\left\langle O_{k} \Psi_{1}(T, \epsilon+Y), \Psi_{1}(T, \epsilon+Y)\right\rangle=\mathcal{L}_{Y}\left\langle O_{k} \Psi_{2}(T, \epsilon+Y), \Psi_{2}(T, \epsilon+Y)\right\rangle \quad \forall \epsilon \in L^{1}([0, T] ; \mathbb{R}), \quad \forall k=1, \ldots, K,
$$

then either the conclusion (4.5) or the conclusion (4.6) of the Theorem 4.6 holds (see also Rem. 4.8).

Remark 5.3. When $\mathcal{O}$ is not a CSCO, the same proof allows only to obtain that an isomorphism of Lie algebras exists that sends $i H_{1}$ to $i H_{2}$ and $i \mu_{1}$ to $i \mu_{2}$. 
Remark 5.4. Relation (5.8) does not imply that for any $\gamma_{k} \in \mathbb{R}$ :

$$
\begin{aligned}
& \forall \epsilon \in L^{1}([0, T] ; \mathbb{R}): \\
& \mathcal{L}_{Y}\left\langle\left(\sum_{k=1}^{K} \gamma_{k} O_{k}\right) \Psi_{1}(T, \epsilon+Y), \Psi_{1}(T, \epsilon+Y)\right\rangle=\mathcal{L}_{Y}\left\langle\left(\sum_{k=1}^{K} \gamma_{k} O_{k}\right) \Psi_{2}(T, \epsilon+Y), \Psi_{2}(T, \epsilon+Y)\right\rangle,
\end{aligned}
$$

because the probability laws are not additive. This is in contrast with the situation in the Theorem 4.6 (see Eqs. (4.11) and (4.12)). But the relation remains true for any operator of the form $a O_{k}+b I d, a, b \in \mathbb{R}$.

Proof. Choose $m_{0} \in \mathbb{N}$ such that $m_{0} \xi_{1}>1$. Then there exists some $\eta>0$ small enough with $\sum_{m=0}^{m_{0}}\left(\xi_{1}-m \eta\right)>1$ and $\xi_{1}-m_{0} \eta>\eta$. As $\sum_{k \in \mathcal{I}} \xi_{k}=1$, there exists $n_{0}^{\prime} \in \mathcal{I}$ such that $\sum_{k \in \mathcal{I}, k>n_{0}^{\prime}} \xi_{k}<\eta$ (by convention a sum over an empty set of indexes is zero). According to Definition 3.2, for all $\ell, \ell^{\prime} \in\left\{1, \ldots, n_{0}^{\prime}\right\}$, if the collection of 2 systems (3.3) for $A_{1}=-i H_{1}+y_{\ell}\left(-i \mu_{1}\right) \in \mathfrak{s u}(N), B_{1}=-i \mu_{1} \in \mathfrak{s u}(N)$ and $A_{2}=-i H_{2}+y_{\ell^{\prime}}\left(-i \mu_{2}\right) \in \mathfrak{s u}(N)$, $B_{2}=-i \mu_{2} \in \mathfrak{s u}(N)$ is simultaneously controllable, then there exists $T_{H_{1}, H_{2}, \mu_{1}, \mu_{2}, y_{\ell}, y_{\ell^{\prime}}}>0$ such that the collection is simultaneously controllable at all times $T \geq T_{H_{1}, H_{2}, \mu_{1}, \mu_{2}, y_{\ell}, y_{\ell^{\prime}}}$. If the collection is not controllable, we take $T_{H_{1}, H_{2}, \mu_{1}, \mu_{2}, y_{\ell}, y_{\ell^{\prime}}}$ to be the time required to control one system (to any target). According to Theorem 3.5, we know that the collection of $n_{0}^{\prime}$ systems (3.4) with $A=-i H_{2}, B=-i \mu_{2}$ and $\left(\alpha_{1}, \ldots, \alpha_{n_{0}^{\prime}}\right)=\left(y_{1}, \ldots, y_{n_{0}^{\prime}}\right)$ is simultaneously controllable therefore there exists $T_{H_{2}, \mu_{2}, y_{1}, \ldots, y_{n_{0}^{\prime}}}$ such that the collection is simultaneously

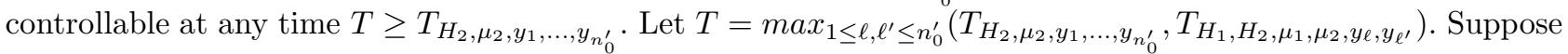
that the observations follow the same law at time $T$. Recall that $\Psi_{a}\left(T, \epsilon+y_{\ell}\right)=X_{a}\left(T, \epsilon+y_{\ell}\right) \Psi_{0}^{a}$ with $X_{a}\left(t, \epsilon+y_{\ell}\right)$ solutions of (5.1) where $A_{a}=-i H_{a}, B_{a}=-i \mu_{a}$, for $a=1,2$ respectively and $h\left(\epsilon(t), y_{\ell}\right)=\epsilon(t)+y_{\ell}$.

The second part of Remark 5.4 implies that we can suppose, without loss of generality, that any $\tilde{O} \in \mathcal{O}$ has the smallest eigenvalue equal to 0 and the largest one equal to 1 . Fix now $\tilde{O} \in \mathcal{O}$. We apply the Lemma 5.1 to $X \mapsto\left\langle\tilde{O} X \Psi_{0}^{a}, X \Psi_{0}^{a}\right\rangle, a=1,2$ which are obviously analytic with respect to $X$. Then for all $\ell \in \mathcal{I}, \exists \kappa(\ell)$ such that

$$
\left\langle\tilde{O} \Psi_{1}\left(T, \epsilon+y_{\ell}\right), \Psi_{1}\left(T, \epsilon+y_{\ell}\right)\right\rangle=\left\langle\tilde{O} \Psi_{2}\left(T, \epsilon+y_{\kappa(\ell)}\right), \Psi_{2}\left(T, \epsilon+y_{\kappa(\ell)}\right)\right\rangle .
$$

Recall equation (5.4) in Lemma 5.1 (we use the same notations):

$$
\sum_{k \in \mathcal{I} / v_{1}^{k}=v_{1}^{\ell}} \xi_{k}=\sum_{k^{\prime} \in \mathcal{I} / v_{2}^{k^{\prime}}=v_{1}^{\ell}} \xi_{k^{\prime}}
$$

for any control $\epsilon(t) \in \mathrm{L}^{1}([0, T], \mathbb{R})$. Now let us take $\ell=1$. In Lemma 5.1 we proved that $\kappa(1) \leq n_{0}(1)$ with $\sum_{k>n_{0}(1)-1} \xi_{k} \geq \xi_{1}>\xi_{1}-m_{0} \eta>\eta>\sum_{k>n_{0}^{\prime}} \xi_{k}$. Thus $n_{0}^{\prime} \geq n_{0}(1) \geq \kappa(1)$. By simultaneous controllability, there exists a control $\epsilon$ such that $\left\langle\tilde{O} \Psi_{2}\left(T, \epsilon+y_{\kappa(1)}\right), \Psi_{2}\left(T, \epsilon+y_{\kappa(1)}\right)\right\rangle=1$ and $\left\langle\tilde{O} \Psi_{2}\left(T, \epsilon+y_{j}\right), \Psi_{2}\left(T, \epsilon+y_{j}\right)\right\rangle=0$ for all $j \leq n_{0}^{\prime}$ and $j \neq \kappa(1)$. In addition, Lemma 5.1 proves that for any control $\epsilon, v_{1}^{1}=v_{2}^{\kappa(1)}=1$. So for this $\epsilon$,

$$
\xi_{1} \leq \sum_{k \in \mathcal{I} / v_{1}^{k}=v_{2}^{\kappa(1)}} \xi_{k}=\sum_{k^{\prime} \in \mathcal{I} / v_{2}^{k^{\prime}}=v_{2}^{\kappa(1)}} \xi_{k^{\prime}} \leq \xi_{\kappa(1)}+\sum_{k>n_{0}^{\prime}} \xi_{k} \leq \xi_{\kappa(1)}+\eta .
$$

We deduce that $\xi_{\kappa(1)} \geq \xi_{1}-\eta$. With the same reasoning and by recurrence we demonstrate that $\xi_{\kappa^{m}(1)} \geq \xi_{1}-m \eta$ for any $m \in\left\{1, \ldots, m_{0}\right\}$ thanks to the relationship $\sum_{k>n_{0}\left(\kappa^{m}(1)\right)-1} \xi_{k} \geq \xi_{\kappa^{m}(1)} \geq \xi_{1}-m \eta \geq \xi_{1}-m_{0} \eta>\eta>$ $\sum_{k>n_{0}^{\prime}} \xi_{k}$. If $1, \kappa(1), \ldots, \kappa^{m_{0}}(1)$ are all distinct, then $1=\sum_{k \in \mathcal{I}} \xi_{k} \geq \sum_{m=0}^{m_{0}} \xi_{\kappa^{m}(1)}>1$, which leads to a contradiction. So at least two among the $1, \kappa(1), \ldots, \kappa^{m_{0}}(1)$ are equal.

On the other hand equation (5.10) implies that the collection of the two systems

$$
\left\{\begin{array}{l}
\frac{\mathrm{d} X_{1}(t, \epsilon)}{\mathrm{d} t}=\left[-i\left(H_{1}+y_{\kappa^{m}(1)} \mu_{1}\right)+\epsilon(t)\left(-i \mu_{1}\right)\right] X_{1}(t, \epsilon) \\
X_{1}(0, \epsilon)=I d
\end{array}\right.
$$


and

$$
\left\{\begin{array}{l}
\frac{\mathrm{d} X_{2}(t, \epsilon)}{\mathrm{d} t}=\left[-i\left(H_{2}+y_{\kappa^{m+1}(1)} \mu_{2}\right)+\epsilon(t)\left(-i \mu_{2}\right)\right] X_{2}(t, \epsilon) \\
X_{2}(0, \epsilon)=I d
\end{array}\right.
$$

is not ensemble controllable for all $m \in\left\{0, \ldots, m_{0}-1\right\}$. Applying Theorem 3.3 and Lemma 3.4 to $G=S U(N)$, $A_{1}=-i\left(H_{1}+y_{\kappa^{m}(1)} \mu_{1}\right), A_{2}=-i\left(H_{2}+y_{\kappa^{m+1}(1)} \mu_{2}\right), B_{1}=-i \mu_{1}$ and $B_{2}=-i \mu_{2}$ there exist $f_{m}$ automorphisms of $\mathfrak{s u}(N)$ such that $f_{m}\left(-i\left(H_{1}+y_{\kappa^{m}(1)} \mu_{1}\right)\right)=-i\left(H_{2}+y_{\kappa^{m+1}(1)} \mu_{2}\right)$ and $f_{m}\left(-i \mu_{1}\right)=-i \mu_{2}$. By linearity of $f_{1}$ and $f_{m}$, we obtain $\left(f_{m}^{-1} \circ f_{1}\right)\left(-i H_{1}\right)=-i H_{1}+\left[\left(y_{\kappa(1)}-y_{1}\right)-\left(y_{\kappa^{m+1}(1)}-y_{\kappa^{m}(1)}\right)\right]\left(-i \mu_{1}\right)$ and $\left(f_{m}^{-1} \circ f_{1}\right)\left(-i \mu_{1}\right)=-i \mu_{1}$. Denote $f=f_{m}^{-1} \circ f_{1}$ and $\beta=\left(y_{\kappa(1)}-y_{1}\right)-\left(y_{\kappa^{m+1}(1)}-y_{\kappa^{m}(1)}\right)$, then we have $-i H_{1}=f\left(-i H_{1}\right)+i \beta \mu_{1}=$ $f\left(f\left(-i H_{1}\right)+i \beta \mu_{1}\right)+i \beta \mu_{1}=f^{2}\left(-i H_{1}\right)+2 i \beta \mu_{1}$ and by recurrence $-i H_{1}=f^{p}\left(-i H_{1}\right)+i p \beta \mu_{2}$ for all $p \in \mathbb{N}$. All automorphisms of $\mathfrak{s u}(N)$ belong to a compact set hence the set $\left\{f^{p}\left(-i H_{1}\right) \in \mathfrak{s u}(N), p \geq 0\right\}$ is bounded for all $m$. Therefore the sequence $\left(i p \beta \mu_{2}\right)_{p \geq 0}$ is bounded which implies $\beta \mu_{2}=0$. According to assumption (A1), $\mu_{2} \neq 0$. Thus $\beta=0$. Denote $C=y_{\kappa(1)}-y_{1}$, then $y_{\kappa^{m+1}(1)}=y_{\kappa^{m}(1)}+C \forall m \in\left\{0, \ldots, m_{0}-1\right\}$. As $1, \kappa(1), \ldots, \kappa^{m_{0}}(1)$ are not all different, $C=0$.

Since $\tilde{O} \in \mathcal{O}$ was arbitrary we proved so far that the systems without noise $\left(H_{1}+y_{1} \mu_{1}, \mu_{1}\right)$ and $\left(H_{2}+y_{1} \mu_{2}, \mu_{2}\right)$ give the same observations for the $\operatorname{CSCO} \mathcal{O}$; the conclusion follows from the Theorem 4.6.

Remark 5.5. Here and in all similar results, the time $T$ should be understood as 'if the time is large enough': the proof can be trivially adapted to treat the situation when the equality in law holds at some other final time $T^{*}$ provided that $T^{*}$ is larger than the time $T$ given by the theorem.

A similar reasoning allows to prove for the setting (S1) the following:

Corollary 5.6. Consider the same setting and assumptions as in Theorem 4.1 with the exception of the relation (4.1). Then there exists $T>0$ such that if:

$$
\mathcal{L}_{Y}\left\langle O \Psi_{1}(T, \epsilon+Y), \Psi_{1}(T, \epsilon+Y)\right\rangle=\mathcal{L}_{Y}\left\langle O \Psi_{2}(T, \epsilon+Y), \Psi_{2}(T, \epsilon+Y)\right\rangle \quad \forall \epsilon \in L^{1}([0, T] ; \mathbb{R}), \quad \forall O \in \mathcal{O},
$$

then the conclusion (4.2) of the Theorem 4.1 holds.

\subsection{The multiplicative perturbation case}

In this section we consider the multiplicative perturbation, which means the control is in the form of $u=Y \cdot \epsilon$. We suppose moreover that this perturbation is positive: $\mathcal{V} \subset \mathbb{R}^{+}$.

Corollary 5.7. Consider the same setting and assumptions as in the Theorem 4.6 with the exception of the relation (4.4). Then there exists $T>0$ such that if:

$$
\mathcal{L}_{Y}\left\langle O_{k} \Psi_{1}(T, \epsilon Y), \Psi_{1}(T, \epsilon Y)\right\rangle=\mathcal{L}_{Y}\left\langle O_{k} \Psi_{2}(T, \epsilon Y), \Psi_{2}(T, \epsilon Y)\right\rangle \quad \forall \epsilon \in L^{1}([0, T] ; \mathbb{R}), \quad \forall k=1, \ldots, K,
$$

then either the conclusion (4.5) or the conclusion (4.6) of the Theorem 4.6 holds (see also Rem. 4.8).

Proof. The proof is similar with the exception that the simultaneous controllability result to be used is Corollary 5 , page 25 in [3].

Remark 5.8. When $\mathcal{V}$ also contains negative values, a similar result can be stated. The only difference is that one obtains:

$$
\left\langle\mu_{1} \phi_{j}, \phi_{k}\right\rangle= \pm \mathrm{e}^{i\left(\alpha_{j}-\alpha_{k}\right)}\left\langle\mu_{2} \phi_{j}, \phi_{k}\right\rangle,\left\langle H_{1} \phi_{j}, \phi_{k}\right\rangle= \pm \mathrm{e}^{i\left(\alpha_{j}-\alpha_{k}\right)}\left\langle H_{2} \phi_{j}, \phi_{k}\right\rangle,\left\langle\Psi_{0}^{1}, \phi_{j}\right\rangle= \pm \mathrm{e}^{i\left(\theta-\alpha_{j}\right)}\left\langle\Psi_{0}^{2}, \phi_{j}\right\rangle,
$$

and a similar relation for the conjugate case. Furthermore, the polynomial situation $\mathcal{V} \subset \mathbb{R}^{d}$ with $d>1$, $u(t)=\sum_{a=0}^{d} Y_{a} \epsilon^{a}(t)$ can be studied. But although this case is also tractable with the controllability result in [20], the conclusion is very cumbersome to formulate and we leave it as an exercise for the reader. 
TABLE 1. Law of $Y$ for the numerical example in Section 6. Here $L_{0}=10$; the second row presents the values $y_{\ell}, \ell \leq L_{0}$ which have been chosen randomly (uniformly) in $\left[0,0.1 * \frac{\|H\|_{l} \infty}{\|\mu\|_{l} \infty}=\right.$ 0.0012]. The third row displays the probabilities $\xi_{\ell}, \ell \leq L_{0}$ which have been chosen at random, uniformly in $[0,1]$, the sum rescaled to 1 and then ordered such that $\left(\xi_{\ell}\right)_{\ell \geq 1}$ is a decreasing sequence.

\begin{tabular}{ccccccccccc}
\hline$\ell$ & 1 & 2 & 3 & 4 & 5 & 6 & 7 & 8 & 9 & 10 \\
\hline$y_{\ell}$ & 0.000400 & 0.000066 & 0.001025 & 0.000224 & 0.000816 & 0.000679 & 0.000740 & 0.000975 & 0.000211 & 0.000156 \\
$\xi_{\ell}$ & 0.181810 & 0.163630 & 0.145450 & 0.127270 & 0.109090 & 0.090900 & 0.072720 & 0.054540 & 0.036360 & 0.018180 \\
\hline
\end{tabular}

\section{Numerical APPLiCATion}

Numerical tests are presented for the setting of the Theorem 5.2. We consider the 4-level system $(N=4)$ in [8] and want to recover the Hamiltonian matrix $H_{\text {real }}$ and the dipole moment matrix $\mu_{\text {real }}$ :

$$
H_{\text {real }}=\left(\begin{array}{cccc}
0.0833 & -0.0038 & -0.0087 & 0.0041 \\
-0.0038 & 0.0647 & 0.0083 & 0.0038 \\
-0.0087 & 0.0083 & 0.0036 & -0.0076 \\
0.0041 & 0.0038 & -0.0076 & 0.0357
\end{array}\right), \quad \mu_{\text {real }}=\left(\begin{array}{cccc}
0 & 5 & -1 & 0 \\
5 & 0 & 6 & -1.5 \\
-1 & 6 & 0 & 7 \\
0 & -1.5 & 7 & 0
\end{array}\right) .
$$

Note that:

$$
H_{\text {real }}=\mathrm{e}^{\mathcal{P}_{\text {real }}} D \mathrm{e}^{-\mathcal{P}_{\text {real }}}, \quad D=\left(\begin{array}{cccc}
0 & 0 & 0 & 0 \\
0 & 0.0365 & 0 & 0 \\
0 & 0 & 0.0651 & 0 \\
0 & 0 & 0 & 0.0857
\end{array}\right), \mathcal{P}_{\text {real }}=\left(\begin{array}{cccc}
0 & 1 & -1 & 1 \\
-1 & 0 & 1 & 1 \\
1 & -1 & 0 & -1 \\
-1 & -1 & 1 & 0
\end{array}\right)
$$

In practice the eigenvalues of the free Hamiltonian are measured by spectrometry and hence known with high precision, see also the discussion in ([16], p. 379 and Rem. 7, p. 384). Accordingly, we suppose that the eigenvalues of $H_{\text {real }}$ are known i.e., the matrix $D$ is known. So identifying $H_{\text {real }}$ is equivalent to identifying the anti-Hermitian rotation matrix $\mathcal{P}_{\text {real }}$.

The law of the perturbation $Y$ is given in Table 1. We consider a finite set of test control fields of the form:

$$
\epsilon(t)=\exp \left(-40(t-T / 2)^{2} / T^{2}\right) \sum_{i<j, i, j=1}^{N} A_{i, j} \sin \left[\left(\lambda_{j}\left(H_{\text {real }}\right)-\lambda_{i}\left(H_{\text {real }}\right)\right) t+\theta_{i, j}\right] .
$$

Here $\lambda_{i}\left(H_{\text {real }}\right)$ are eigenvalues of $H_{\text {real }}, i \leq N$ and $A_{i, j}, \theta_{i, j}$ are parameters to be chosen later. The total simulation time is $T=3200$ which means about 10 periods of the smallest transition frequency $\lambda_{4}\left(H_{\text {real }}\right)-$ $\lambda_{3}\left(H_{\text {real }}\right)$.

Let $\left\{e_{k} ; k \leq N\right\}$ be the canonical basis of $\mathbb{C}^{N}$ and $\mathcal{O}=\left\{e_{k} e_{k}^{*}, k \leq N\right\}$ (populations).

We choose $N_{\epsilon}=36$ controls $\epsilon_{1}(t), \ldots, \epsilon_{N_{\epsilon}}(t)$ drawing $\theta_{i j}$ uniformly in $[0,2 \pi]$ and $A_{i j}$ uniformly in $[0,0.0012]$ and we define the functional to be minimized:

$$
\mathcal{J}(\mathcal{P}, \mu)=\sum_{i=1}^{N_{\epsilon}} \sum_{j=1}^{N} d_{\mathcal{W}_{1}}\left(\mathcal { L } _ { Y } \left(\left|\left\langle\Psi\left(T, \mathrm{e}^{\mathcal{P}} D \mathrm{e}^{-\mathcal{P}}, \epsilon_{i}+Y, \mu, \Psi_{1}^{0}\right), e_{j}\right\rangle\right|^{2}, \mathcal{L}_{Y}\left(\left|\left\langle\Psi\left(T, H_{\text {real }}, \epsilon_{i}+Y, \mu_{\text {real }}, \Psi_{\text {real }}^{0}\right), e_{j}\right\rangle\right|^{2}\right) .\right.\right.
$$

Here we use the 1-Wasserstein distance (see pp. 34-35 in [22]) $d_{\mathcal{W}_{1}}$ between two laws $L_{Y} Z_{1}$ and $L_{Y} Z_{2}$ defined as $d_{\mathcal{W}_{1}}\left(L_{Y} Z_{1}, L_{Y} Z_{2}\right)=\int_{0}^{1}\left|F_{Z_{1}}^{-1}(x)-F_{Z_{2}}^{-1}(x)\right| \mathrm{d} x$ with $F_{Z_{1}}$ (respectively $F_{Z_{2}}$ ) the cumulative distribution function of $Z_{1}$ (respectively $Z_{2}$ )(see pp. $73-75$ in [22] for details). We start with $10 \%$ relative error on $\mu$ and $\mathcal{P}$ and we use 

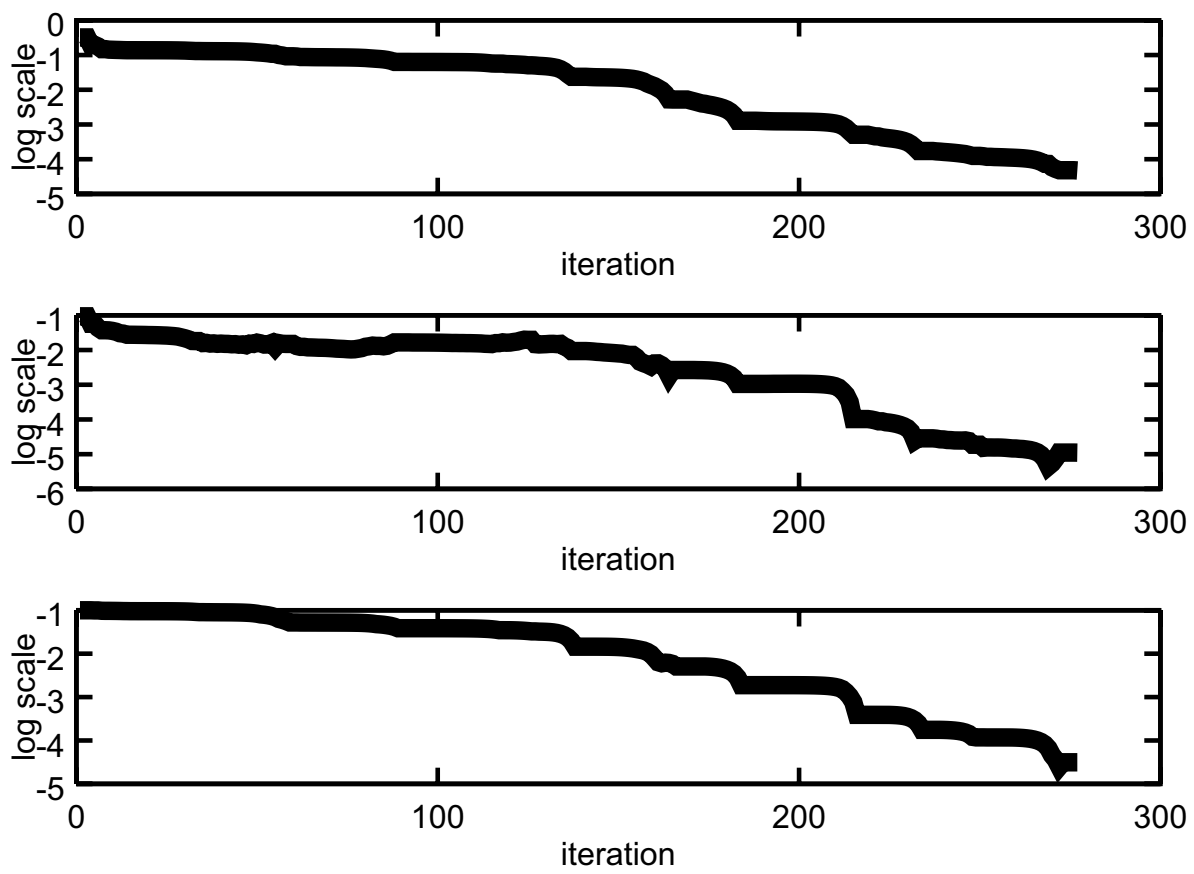

FiguRE 1. The (base 10) logarithm of $\mathcal{J}$ (upper plot), the (base 10) logarithm of the relative error on $\mathcal{P}$ (middle plot) and the (base 10) logarithm of the relative error on $\mu$ (lower plot) as a function of the iteration index.

a classical unconstrained nonlinear optimization algorithm to minimize $\mathcal{J}(\mathcal{P}, \mu)$ (we used the Gnu Octave $[9,10]$ procedure "fminunc"). After 277 iterations, we find:

$$
\mathcal{P}_{277}=\left(\begin{array}{cccc}
0 & 0.999 & -0.999 & 1.002 \\
-0.999 & 0 & 1 & 0.999 \\
0.999 & -1 & 0 & -1.002 \\
-1.002 & -0.999 & 1.002 & 0
\end{array}\right), \quad \mu_{277}=\left(\begin{array}{cccc}
0 & 4.999 & -0.998 & -0.003 \\
4.999 & 0 & 6 & -1.5 \\
-0.998 & 6 & 0 & 7 \\
-0.003 & -1.5 & 7 & 0
\end{array}\right) \text {. }
$$

This corresponds to $0.003 \%$ relative error on $\mu$ and $0.001 \%$ relative error on $\mathcal{P}$. We note that the histograms for $\left(\mathcal{P}_{\text {real }}, \mu_{\text {real }}\right)$ and $\left(\mathcal{P}_{277}, \mu_{277}\right)$ are nearly the same. See Figures 1 and 2 for details.

\section{Perspectives And CONCLUding REMARKS}

Among the limitations of the present work is the requirement to consider only time-independent perturbations; it would be interesting to consider time-dependent perturbations and more elaborate noise models (beyond polynomial) and, of course, perturbations that can take values in an uncountable set (in the same spirit as in $[2,17])$. Extension to infinite dimensional quantum systems can also be interesting; in all these cases one technical limitation is the absence of simultaneous controllability results analogue to Theorems 3.3 and 3.5, still missing in general even for finite dimensional models as soon as the dimension is larger than 4.

A distinct extension, which seems attainable with the tools presented here, is to consider a framework that involves density matrices instead of wave-functions.

Acknowledgements. The authors acknowledge support from the Agence Nationale de la Recherche (ANR), Projet Blanc EMAQS No. ANR-2011-BS01-017-01. 

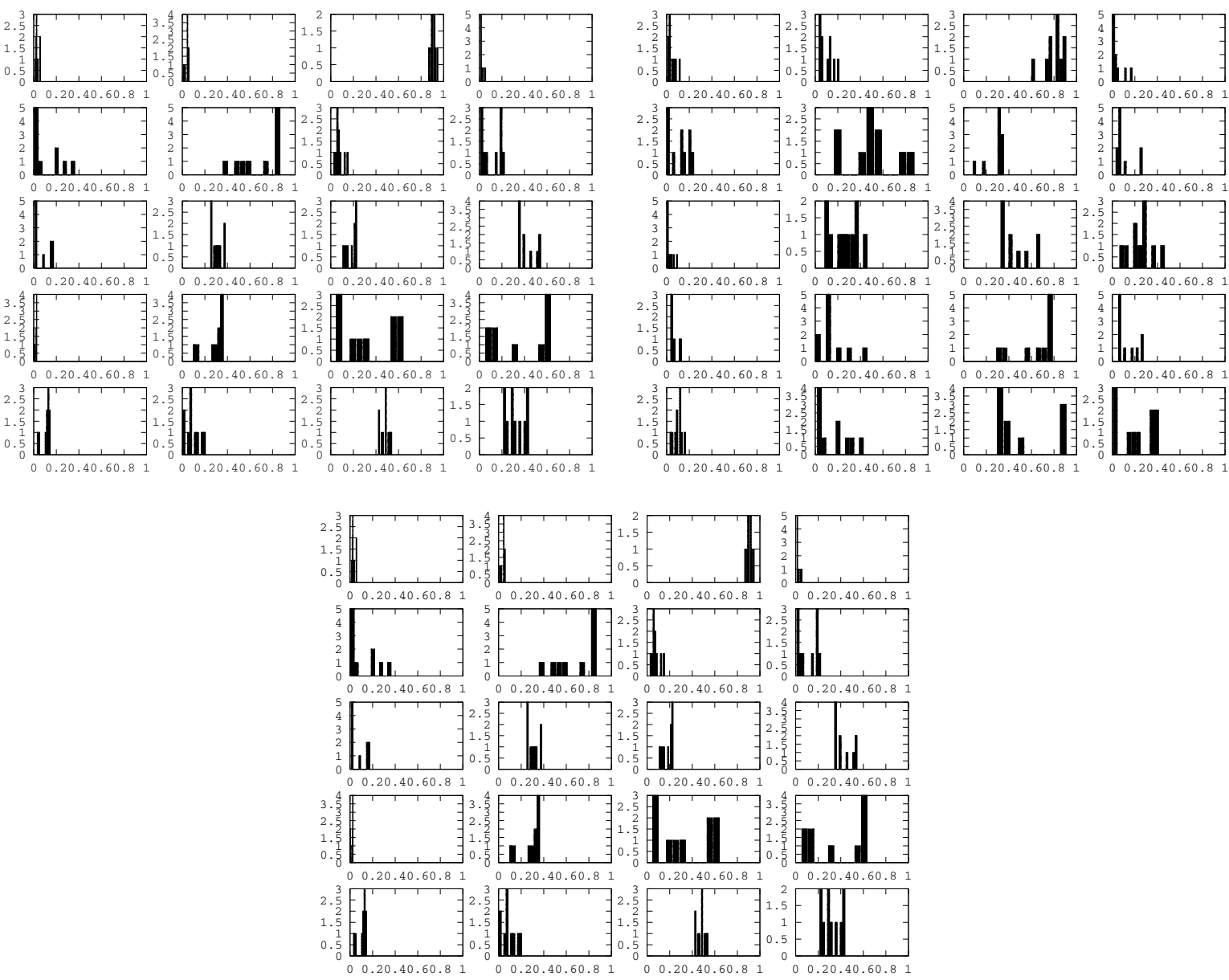

FiguRE 2. The optimization algorithm iterates starting from the initial guess $\left(\mathcal{P}_{0}, \mu_{0}\right)$ and constructs a sequence of estimations $\left(\mathcal{P}_{k}, \mu_{k}\right)$. We plot the histograms of the laws $\mathcal{L}_{Y}\left(\left|\left\langle\Psi\left(T, \mathrm{e}^{\mathcal{P}_{k}} D \mathrm{e}^{-\mathcal{P}_{k}}, \epsilon_{i}+Y, \mu_{k}, \Psi_{1}^{0}\right), e_{j}\right\rangle\right|^{2}\right.$ for various choices of $k, i=1, \ldots, 5$ and $j=1,2,3,4$. In the top picture are the histograms of the observations $\left(\mathcal{P}_{\text {real }}, \mu_{\text {real }}\right)$, in the bottom left picture are the histograms for the initial guess $\left(\mathcal{P}_{0}, \mu_{0}\right)(k=0)$ and in the bottom right image the histograms for the final iteration $\left(\mathcal{P}_{277}, \mu_{277}\right)(k=277)$. The optimization works well as there is an obvious match between the top and the bottom right histograms.

\section{REFERENCES}

[1] L. Baudouin and A. Mercado, An inverse problem for Schrodinger equations with discontinuous main coefficient. Applicable Analysis 87 (2008) 1145-1165.

[2] K. Beauchard, J.-M. Coron, and P. Rouchon, Controllability issues for continuous-spectrum systems and ensemble controllability of Bloch equations. Comm. Math. Phys. 296 (2010) 525-557.

[3] M. Belhadj, J. Salomon, and G. Turinici, Ensemble controllability and discrimination of perturbed bilinear control systems on connected, simple, compact Lie groups. Eur. J. Control 22 (2015) 23-29.

[4] S. Bonnabel, M. Mirrahimi, and P. Rouchon, Observer-based Hamiltonian identification for quantum systems. Automatica 45 (2009) 1144-1155.

[5] C. Brif, R. Chakrabarti, and H. Rabitz, Control of quantum phenomena: past, present and future. New J. Phys. 12 (2010) 075008 .

[6] C. Cohen-Tannoudji, B. Diu and F. Laloë, Quantum Mechanics, Vol 1. Wiley, New-York (1977). 
[7] D. D'Alessandro, Introduction to quantum control and dynamics. Chapman 8 Hall/CRC Applied Mathematics and Nonlinear Science Series. Chapman \& Hall/CRC, Boca Raton, FL (2008).

[8] A. Donovan and H. Rabitz, Exploring the Hamiltonian inversion landscape. Phys. Chem. Chem. Phys. 16 (2014) 15615-15622.

[9] J.W. Eaton, D. Bateman, S. Hauberg and R. Wehbring, GNU Octave version 4.0.0 manual: a high-level interactive language for numerical computations. Available at http://www.gnu.org/software/octave/doc/interpreter (2015).

[10] J.W. Eaton et al., GNU Octave 4.0.0. Available at http://www.octave.org (2015).

[11] J.M. Geremia and H. Rabitz, Optimal Hamiltonian identification: The synthesis of quantum optimal control and quantum inversion. J. Chem. Phys. 118 (2003) 5369-5382.

[12] D. Hocker, Co. Brif, M.D. Grace, A. Donovan and T.-S. Ho, K. Moore Tibbetts, R. Wu and H. Rabitz, Characterization of control noise effects in optimal quantum unitary dynamics. Phys. Rev. A 90 (2014) 062309.

[13] V. Jurdjevic and H.J. Sussmann. Control systems on Lie groups. J. Differ. Eq. 12 (1972) 313-329.

[14] K. Khodjasteh and L. Viola, Dynamical quantum error correction of unitary operations with bounded controls. Phys. Rev. A 80 (2009) 032314.

[15] K. Khodjasteh and L. Viola, Dynamically error-corrected gates for universal quantum computation. Phys. Rev. Lett. 102 (2009) 080501.

[16] C. Le Bris, M. Mirrahimi, H. Rabitz and G. Turinici, Hamiltonian identification for quantum systems: well-posedness and numerical approaches. ESAIM: COCV 13 (2007) 378-395.

[17] J.-S. Li and N. Khaneja, Control of inhomogeneous quantum ensembles. Phys. Rev. A 73 (2006) 030302.

[18] Y. Maday and J. Salomon, A greedy algorithm for the identification of quantum systems. In Decision and Control, 2009 held jointly with the 2009 28th Chinese Control Conference. Proc. of the 48th IEEE Conference on CDC/CCC 2009 (2009) $375-379$.

[19] A.M. Souza, G.A. Álvarez and D. Suter, Experimental protection of quantum gates against decoherence and control errors. Phys. Rev. A 86 (2012) 050301.

[20] G. Turinici, Beyond bilinear controllability: applications to quantum control. In Control of coupled partial differential equations, Vol. 155 of Internat. Ser. Numer. Math. Oberwolfach, Allemagne. Birkhauser (2007) 293-309.

[21] G. Turinici, V. Ramakhrishna, B. Li and H. Rabitz, Optimal discrimination of multiple quantum systems: controllability analysis. J. Phys. A 37 (2004) 273.

[22] C. Villani, Topics in optimal transportation. Graduate Studies in Mathematics. American Mathematical Society, cop., Providence R.I. (2003).

[23] W. Zhu and H. Rabitz, Potential surfaces from the inversion of time dependent probability density data. J. Chem. Phys. 111 (1999) 472-480.

[24] I.R. Zola and H. Rabitz, The influence of laser field noise on controlled quantum dynamics. J. Chem. Phys. 120 (2004) 9009-9016. 